\title{
Prenatal exposure to cigarette smoke or alcohol and cerebellum volume in attention-deficit/hyperactivity disorder and typical development
}

\author{
P de Zeeuw, F Zwart, R Schrama, H van Engeland and S Durston
}

Prenatal exposure to teratogenic substances, such as nicotine or alcohol, increases the risk of developing attention-deficit/ hyperactivity disorder (ADHD). To date, studies examining this relationship have used symptom scales as outcome measures to assess the effect of prenatal exposure, and have not investigated the neurobiological pathways involved. This study explores the effect of prenatal exposure to cigarettes or alcohol on brain volume in children with ADHD and typically developing controls. Children with ADHD who had been exposed prenatally to either substance were individually matched to children with and without ADHD who had not been. Controls who had been exposed prenatally were also individually matched to controls who had not been. For prenatal exposure to both smoking and alcohol, we found a pattern where subjects with ADHD who had been exposed had the smallest brain volumes and unexposed controls had the largest, with intermediate volumes for unexposed subjects with ADHD. This effect was most pronounced for cerebellum. A similar reduction fell short of significance for controls who had been exposed to cigarettes, but not alcohol. Our results are consistent with an additive effect of prenatal exposure and ADHD on brain volume, with the effects most pronounced for cerebellum.

Translational Psychiatry (2012) 2, e84; doi:10.1038/tp.2012.12; published online 6 March 2012

\section{Introduction}

Prenatal exposure to teratogenic substances, such as nicotine and alcohol, is associated with long-term negative effects on cognition and behavior. ${ }^{1-6}$ One behavioral sequel is an increased risk of developing attention-deficit/hyperactivity disorder (ADHD). In fact, exposure to these substances is currently one of the more vigorously investigated environmental risk factors for ADHD. ${ }^{3,5,7,8}$ The biological mechanisms underlying the association may be diverse ${ }^{9-11}$ but it is generally assumed that prenatal exposure disrupts early brain development and that its effects continue over development. ${ }^{1,12}$ However, to date studies investigating the association between prenatal exposure to teratogenic substances and ADHD have been based on behavioral ratings and have not assessed the neurobiological substrates underlying the association.

Prenatal maternal cigarette smoking or use of alcohol potentially carries several risks to the developing brain. Both are known to alter placenta functioning indirectly and may cause hypoxic events. ${ }^{1,10,13,14}$ The greatest immediate threat associated with cigarette smoking comes from nicotine, through its interaction with nicotine acetylcholine receptors. ${ }^{1,9-11}$ Acetylcholine is an important regulator of cell survival, neuronal path finding and targeting, migration, and the generation of neural circuits in the developing brain. ${ }^{1,9,15}$ Direct effects of alcohol on prenatal brain development include interference with neurotrophic factors and cell adhesion molecules, altered glial maturation, and dysregulation of cell migration. ${ }^{14,16,17}$ Cell damage and loss may be caused by oxidative stress, excitotoxicity or by apoptosis mediated by glutamergic antagonism. ${ }^{14,16,18}$

In the longer term, the cerebellum may be particularly vulnerable to prenatal exposure to both nicotine and alcohol. The cerebellum shows a protracted developmental pattern, ${ }^{19}$ potentially rendering it vulnerable to the effects of prenatal exposure over a relatively long period. In utero reductions in the size of cerebellum have been reported for fetuses of mothers who smoked during pregnancy. ${ }^{20}$ The developing cerebellum may be also be particularly sensitive to the effects of alcohol exposure, as alcohol has been shown to lead to cell death of Purkinje cells in particular. ${ }^{14,21,22}$

Structural neuroimaging studies have reported brain changes related in both ADHD and prenatal alcohol exposure that show striking similarity, suggesting that prenatal exposure to teratogenic substances may, in some cases, have a role in aberrant brain development in ADHD. Reductions in the volume of the whole brain, cerebellum and caudate are replicated findings in children prenatally exposed to alcohol, ${ }^{13,17}$ and in ADHD. ${ }^{23,24}$ Structural neuroimaging studies of children exposed prenatally to nicotine are scarcer, but those available have reported reductions in volume of similar brain regions, including of total brain, and prefrontal cortex. ${ }^{25-27}$

Behavioral genetic studies have recently suggested that the effect of prenatal exposure to teratogenic substances on the development of behavioral problems may be largely

Neuroimaging Laboratory, Department of Child and Adolescent Psychiatry, Rudolf Magnus Institute of Neuroscience, University Medical Center Utrecht, Utrecht, The Netherlands

Correspondence: Dr P de Zeeuw, Neuroimaging Laboratory, Department of Child and Adolescent Psychiatry, University Medical Center Utrecht/Rudolf Magnus Institute of Neuroscience, Heidelberglaan 100 (HP A-01.468-431), Utrecht, 3584 CX, The Netherlands.

E-mail: p.dezeeuw @umcutrecht.nl

Keywords: ADHD; alcohol; cerebellum; MRI; nicotine; prenatal exposure

Received 25 May 2011; revised 23 January 2012; accepted 25 January 2012 
genetically mediated rather than caused by exposure per se. ${ }^{4,28-30}$ These results could be taken to suggest gene $\times$ environment interaction $(\mathrm{G} \times \mathrm{E})$, but have generally been interpreted as more convergent with $G \times E$ correlations or pleiotropic effects of genes related to both ADHD and substance use. ${ }^{28,30}$ Indeed, the currently available evidence does not suggest a large role for $G \times E, 4,30$ but rather suggests additive effects of risk genes and prenatal exposure. ${ }^{4}$ One important implication of these studies is that they suggest that detecting the effect of an environmental risk factor in isolation is likely to be difficult. ${ }^{29,30}$

This study is a naturalistic study of the effects of selfreported maternal smoking and alcohol use during pregnancy on global brain volumes in children with ADHD and typically developing controls. We compared brain measures derived from anatomical magnetic resonance imaging scans from subjects with ADHD who had been prenatally exposed to cigarettes or alcohol to those from subjects with ADHD who had not, and to those from typically developing controls who also did not have a history of prenatal exposure. Given the results from behavioral genetic studies, we hypothesized a stepwise pattern where we expected the largest volumes for typically developing controls, followed by subjects with ADHD who had not been exposed to teratogenic substances prenatally, and smallest volumes for subjects with ADHD who had. We expected this pattern to be most pronounced for cerebellum, given its hypothesized increased vulnerability to prenatal exposure to teratogenic substances. To investigate the specificity of these results to ADHD, we conducted a second analysis where we compared brain volumes from typically developing controls who had been exposed prenatally to controls who had not. Here, we hypothesized that we would find similar effects of exposure on brain volume, but that these would be attenuated in magnitude compared with the effects for children with ADHD and prenatal exposure.

\section{Participants and methods}

Participants. Participants in this study were selected from a large longitudinal case-control cohort study of brain development in ADHD and typically developing controls including approximately 300 children. The institutional review board approved the study and its procedures. Subjects with ADHD for this cohort were recruited from the outpatient clinic for Disruptive Disorders at the University Medical Center Utrecht, and only after they had received a clinical diagnosis of ADHD. Controls were recruited from elementary schools in the wider Utrecht area. Written informed consent was obtained from parents after full disclosure of the study purpose and procedure. Children provided written and/or verbal assent. The Diagnostic Interview Schedule for Children Version IV (DISC-IV), parent version ${ }^{31}$ was administered to either the mother or to both parents in order to confirm the clinical diagnosis of ADHD of any subtype (ADHD group) or to exclude the presence of any psychiatric morbidity (controls). Parents filled out the Child Behavior Checklist, ${ }^{32}$ to provide a dimensional measure of behavioral symptoms. Controls were excluded if they met criteria for any psychiatric diagnosis or if they had first-degree relatives with a history of psychiatric problems. Children with ADHD were excluded if they met DISC-IV criteria for a co-morbid disorder other than oppositional defiant disorder or conduct disorder. Exclusion of ADHD subjects because of this criterion was rare, as children with more complex clinical presentations including multiple diagnoses were generally not referred to the study. In both groups, additional exclusion criteria were any major physical or neurological disorder or the presence of metals in the body that precluded the magnetic resonance imaging session. Intelligence quotient (IQ) was assessed using the Revised or Third Edition of the Wechsler Intelligence Scale for Children (Dutch version). ${ }^{33}$

At inclusion, parents were sent a questionnaire on family psychiatric history, pregnancy and delivery, which they filled out at home and returned to us via mail or in person during a visit to the department. These questionnaires were typically filled out by the mother. This questionnaire included questions on maternal use of alcohol, cigarettes and other drugs of abuse during pregnancy. The mother was asked whether she had taken the substance in question during pregnancy. If she indicated that she had, she was asked to estimate the number of units (drinks, cigarettes) she had used. Mothers could report the number of units per day, week or month. Estimates were recalculated to number of units per week, which was multiplied by the duration of pregnancy in weeks to acquire an estimate of total use. Birth weight (BW) of the child was also taken from the questionnaire. Questions on the prevalence of psychiatric diagnoses in first-degree family members were used to calculate a proxy for familial psychiatric load. The questionnaires were screened for data quality. This screening preceded the matching procedure and involved checking whether questionnaires were filled out completely and that there were no written comments indicating that parents had not followed the instructions while filling out the questionnaire. Most parents had fully completed the questionnaire. Questionnaires with many missing data or ambiguous answers were reviewed by two of the authors (PdZ and $\mathrm{RS}$ or FZ) and were only carried forward on consensus on the validity of the reported data. For this study, the answers to the questions on substance use during pregnancy were required to be present (77\% met this criterion). For controls, total IQ and mean parental education was slightly lower in the data sets that did not meet this criterion than in those that did (mean parental education M (s.d.) included $=13.5$ (2.0) years, $\mathrm{M}$ (s.d.) not included $=12.3(2.3)$ years; total IQ M (s.d.) $)_{\text {included }}=$ 112.0 (17.0), M (s.d.) not included $=104.0$ (16.4)). No such demographic differences were present for subjects with ADHD.

Alcohol exposure was low in comparison with the extant literature (see Table 1). We set the cutoff for inclusion as a case in the group exposed to alcohol at a minimum of two drinks per month. There were two controls and one case with ADHD for whom prenatal alcohol and cigarette exposure were both reported. Given the small number of cases involved, we included these three subjects in the analyses for both exposures. The mothers of 16 subjects with ADHD (one girl) and 13 controls (two girls) reported cigarette use during pregnancy. The mothers of 11 subjects with ADHD (only boys) and 14 controls (five girls) reported alcohol use during pregnancy. For the individual matching procedure, good 


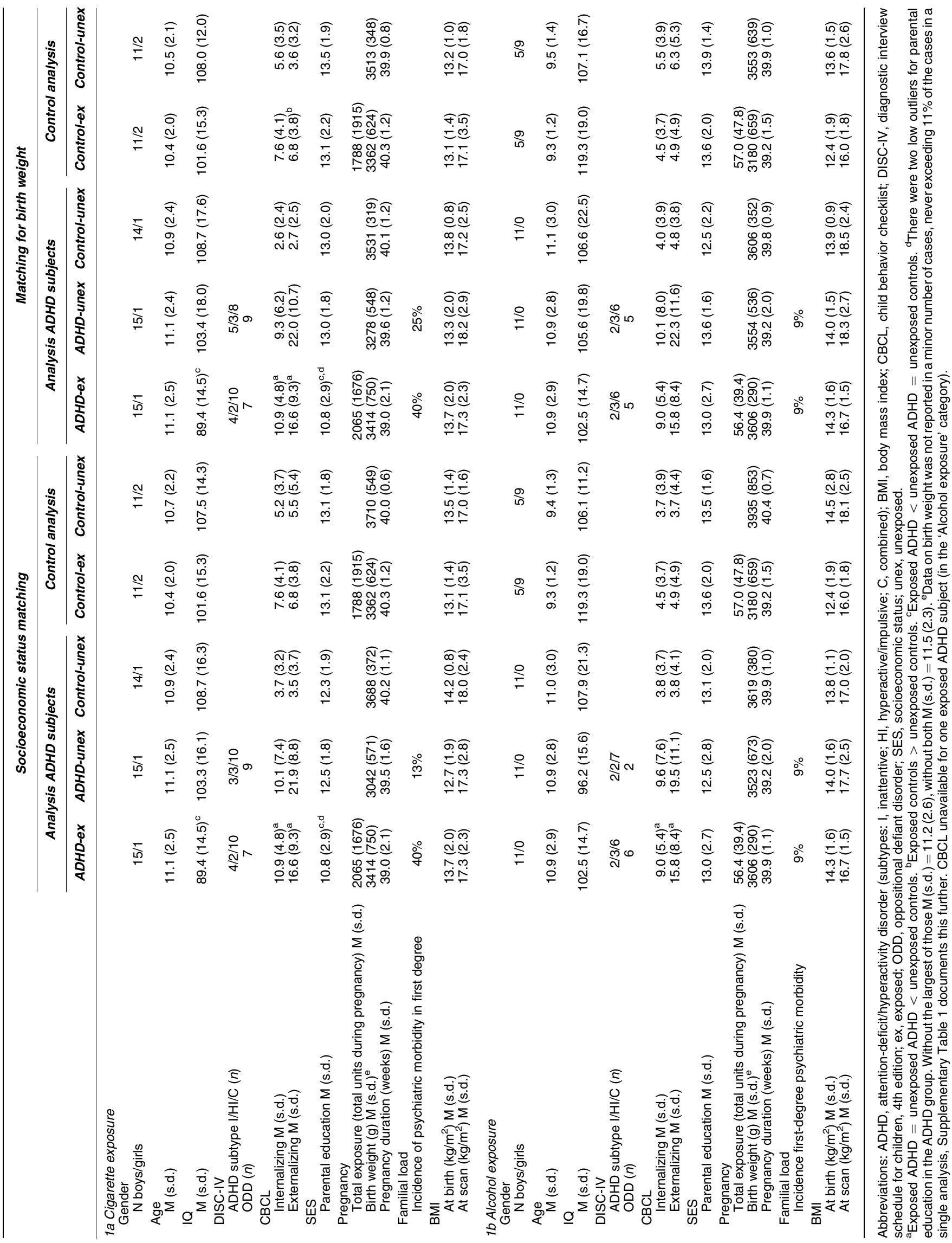


quality questionnaire data were available for 132 unexposed subjects (74 controls, 58 subjects with ADHD).

Matching procedure. Subjects with ADHD and prenatal exposure to either substance were individually matched to subjects with ADHD and typically developing controls who had not been prenatally exposed to either substance from our cohort study. Controls with prenatal exposure were individually matched to controls without. Although a $2 \times 2$ factorial design would have been the design of choice for this study, this was not feasible as we had only limited numbers of subjects with a history of prenatal exposure in the cohort. There were substantial differences in demographic variables between subjects with ADHD and controls who had been exposed for both cigarette and alcohol exposure, prohibiting the use of such a design. Matching was performed blind to medication status of the ADHD participants and to pre- or perinatal complications other than exposure. Subjects were always matched to subjects of the same gender. We performed two rounds of individual matching; one where socioeconomic status (SES) was given priority as a matching criterion and a second where BW was used as the more pressing criterion. First, we matched for SES as this should provide the most parsimonious (if not full) control for confounding issues such as postnatal developmental environment, ${ }^{34}$ and maternal nutritional status, ${ }^{35}$ both of which correlate with SES. The second matching by BW was performed to investigate the specificity of the findings to prenatal exposure, by controlling for general prenatal adversity, for which BW is often considered a proxy.

In the first round of matching, for each prenatally exposed subject, the subject most similar in terms of gender, age at magnetic resonance imaging and SES (mean duration of parental education) was selected from the cohort with the criterion that no prenatal exposure was reported for the latter. In the second round of matching, the same procedure was followed, but a match for BW was given priority over a match for SES. If BW was already optimally matched in the first round (that is, no other subject within the required age range had a smaller difference in BW to the exposed subject), the same subject was retained in the second round of matching. The percentage of changed matches from the first to the second round ranged from $18 \%$ (unexposed controls matched to subjects with ADHD exposed to alcohol) to $50 \%$ (unexposed controls matched to controls exposed to alcohol). A table of both rounds of individual matching is available as Supplementary Material (Supplementary Table 1).

As a result of this individual matching procedure, the groups were highly similar demographically. One notable exception is lower mean IQ for subjects with ADHD who had been prenatally exposed to cigarettes compared with both unexposed subjects with ADHD and the unexposed controls ( $P=0.029$ and $P=0.002$, respectively).

Neuroimaging. All subjects were scanned on a 1.5T Philips MRI scanner (Philips Healthcare, Best, The Netherlands). The imaging protocol and analyses have been previously reported. $^{36-39}$ Briefly, a T1-weighted three-dimensional fast field echo scan of the whole head was acquired with 130 to $1501.5-\mathrm{mm}$ or $1.2-\mathrm{mm}$ contiguous coronal slices. There were no differences in the distribution of 1.2-mm versus 1.5$\mathrm{mm}$ scans between groups in any of the analyses reported.

For the volumetric analysis of the three-dimensional fast field echo scan, a mask of the intracranial space was required. This mask was based on a T2-weighted dual echo turbo spin echo scan with 65 to 75 3.0-mm contiguous coronal slices of the whole head or on the diffusion unweighted volume of a diffusion tensor imaging acquisition during the same session. Previous work has shown that the definition of intracranial volume is comparable using these two methods. ${ }^{39}$ In addition, intracranial masks were manually edited if necessary to ensure accuracy across all scans.

All brain scans were coded to ensure rater blindness to subject identity and diagnosis. The T1 images were automatically placed in Talairach orientation ${ }^{40}$ without scaling, by registering them to a model brain in Talairach orientation. ${ }^{41}$ After linear registration to the T1-weighted image, the intracranial segment served as a mask for all further segmentation steps. The T1-weighted images were corrected for field inhomogeneities using the N3 algorithm. ${ }^{42}$ An automatic image-processing pipeline was used to define the volume of total brain, cerebral and cerebellum volume, and gray matter (GM) and white matter (WM). The software used included updated versions of previously described histogram analysis, mathematical morphology operations and anatomical knowledge based rules to connect all voxels of interest. ${ }^{43,44}$ The gray/white separation algorithm takes into account the effects of partial voluming by calculating the proportion of GM, WM and cerebrospinal fluid for each voxel. ${ }^{45}$ Figure 1 shows a typical GM probability map for cerebellum. Segments for cerebrum and cerebellum were visually checked and edited to ensure an accurate segmentation. Suboptimal scan quality precluded gray/white separation for 4 of 45 children in the analyses for cigarette exposure in ADHD, for 1 of 33 children in the analyses on alcohol exposure in ADHD and for 1 of 28 children in the analyses on control subjects exposed to alcohol.

Statistical analyses. We tested for group differences in demographic data using independent samples $T$-tests, $\chi^{2}$ or Fisher's exact test as appropriate.

The volume of total brain, cerebrum, cerebellum, and GM and WM in cerebrum and cerebellum were compared between groups. For cases where no gray/white separation was possible, the individually matched cases were also removed from the analyses (pairwise removal). Supplementary Table 1 documents four cases where no or suboptimal matching was possible. All analyses were repeated without these cases (based on pairwise removal) with no meaningful effect on any of the results. Therefore, we only report the results with all subjects included.

We used univariate analysis of variance to test for between group differences in volume and performed separate analyses of brain changes related to exposure for subjects with ADHD and controls. A linear polynomial contrast was specified to test our main hypotheses, where exposed subjects with ADHD, unexposed subjects with ADHD and unexposed controls were coded 0,1 and 2, respectively. If the main effect and polynomial were both significant, we conducted post-hoc tests contrasting the exposed to the unexposed subjects with 


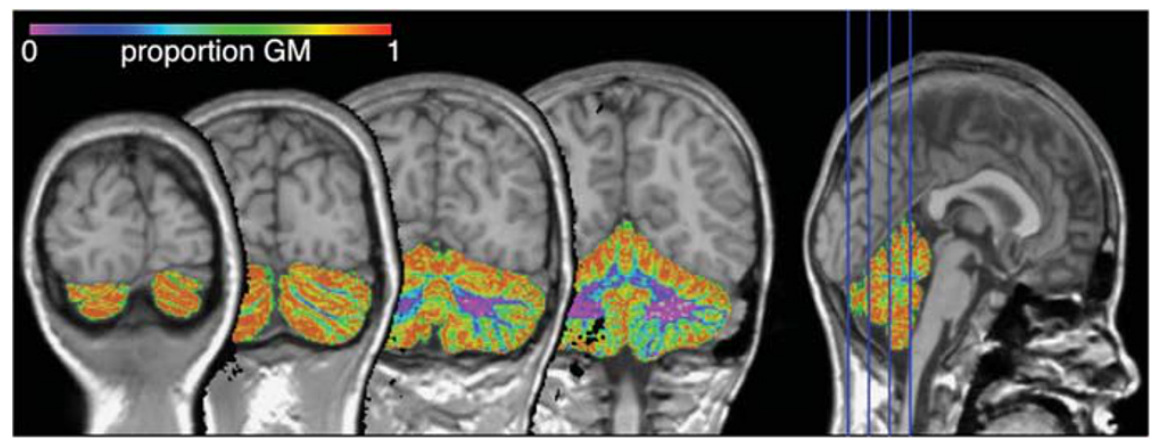

Figure 1 A typical gray/white probability map in cerebellum for a subject in this study. GM, gray matter.

ADHD and the unexposed subjects with ADHD to the unexposed controls. In the analyses of exposed controls, we only tested the main effect of exposure.

As age and gender both have well-established effects on brain volume, ${ }^{46-50}$ we included these parameters as covariates in the models. Furthermore, we included a dummy for scan slice thickness (1.2 versus $1.5 \mathrm{~mm}$ ) as a covariate in all analyses. Analyses were first run for the groups matched by SES and repeated for the groups matched by BW.

\section{Results}

Table 1 lists the group characteristics of the samples matched by SES and BW. There was a small difference in IQ between subjects with ADHD who had been exposed to cigarettes and those who had not. However, there were no correlations between $I Q$ and BW in any of the three subgroups in this analysis. The difference in IQ appeared to be more related to SES, as the only significant correlation was between SES and $I Q$ for subjects with ADHD who had been exposed to cigarettes. This was driven by two outliers with low SES who also had low IQ (72 and 85, respectively).

Table 2 shows the results of the analyses for subjects with ADHD who had been prenatally exposed (separately for nicotine and alcohol exposure). Table 3 shows the results of the analyses for typically developing controls who had been prenatally exposed versus controls who had not.

Cigarette exposure. Although the rate of first-degree psychiatric morbidity was numerically elevated in first-degree relatives of subjects with ADHD who had been exposed to cigarettes compared with those who had not, this difference failed to reach statistical significance (Table 1).

In the analysis of the ADHD group who had been exposed (Table 2, top panel), main effects and significant linear polynomial contrasts were found for total cerebellum volume and for cerebellar GM (Figure 2). For both measures, exposed subjects with ADHD had smaller volumes than unexposed controls, but there was not a significant difference between the unexposed subjects with ADHD and unexposed controls. Results from the same analysis for the groups matched by BW showed a similar pattern of results, with significant main effects and polynomials now found for all brain measures except cerebral WM. Importantly, the means for cerebellum GM volume still showed the same staircase pattern.

Controls who had been exposed showed a trend toward smaller cerebellar WM volume than unexposed controls, but this result fell short of significance $(P=0.057$, Table 3 , top panel). Results from the same analysis matched by BW showed further attenuation of differences between exposed and unexposed controls.

Alcohol exposure. There were no differences in the incidence of psychiatric morbidity in first-degree relatives between the subjects with ADHD who had and had not been exposed to alcohol.

In the analysis for subjects with ADHD (Table 2, bottom panel), we found main effects of group and significant polynomial contrasts for total brain, total cerebral volume and cerebral GM volume and total cerebellum. Again, the pattern was most pronounced and with largest effect size for cerebellum. Post-hoc comparisons showed that cerebellum volume of unexposed subjects with ADHD did not differ from that of unexposed controls, whereas brain volumes of exposed subjects with ADHD did. Figure 3 shows the results for cerebral and cerebellar GM, where unexposed subjects with ADHD show reduced volume compared with unexposed controls for cerebellar GM only. In the analysis for the groups matched by BW, all main effects were no longer significant, with only total cerebellum volume approaching significance $(P=0.057)$ (Table 2).

There were no differences in brain volume between controls who had and had not been exposed to alcohol (Table 3, bottom panel). Results from the same analysis matched by BW were similar (Table 3).

\section{Discussion}

We report that prenatal exposure to both cigarettes and alcohol is related to changes in brain volume. For prenatal exposure to cigarettes, we found a graded pattern, where children with ADHD who had been exposed had the smallest cerebellum volumes, followed by unexposed subjects with $\mathrm{ADHD}$, and the largest volumes in unexposed controls. These results are consistent with separate effects of prenatal exposure and ADHD on cerebellum volume. In controls, a similar effect fell short of statistical significance. For prenatal 


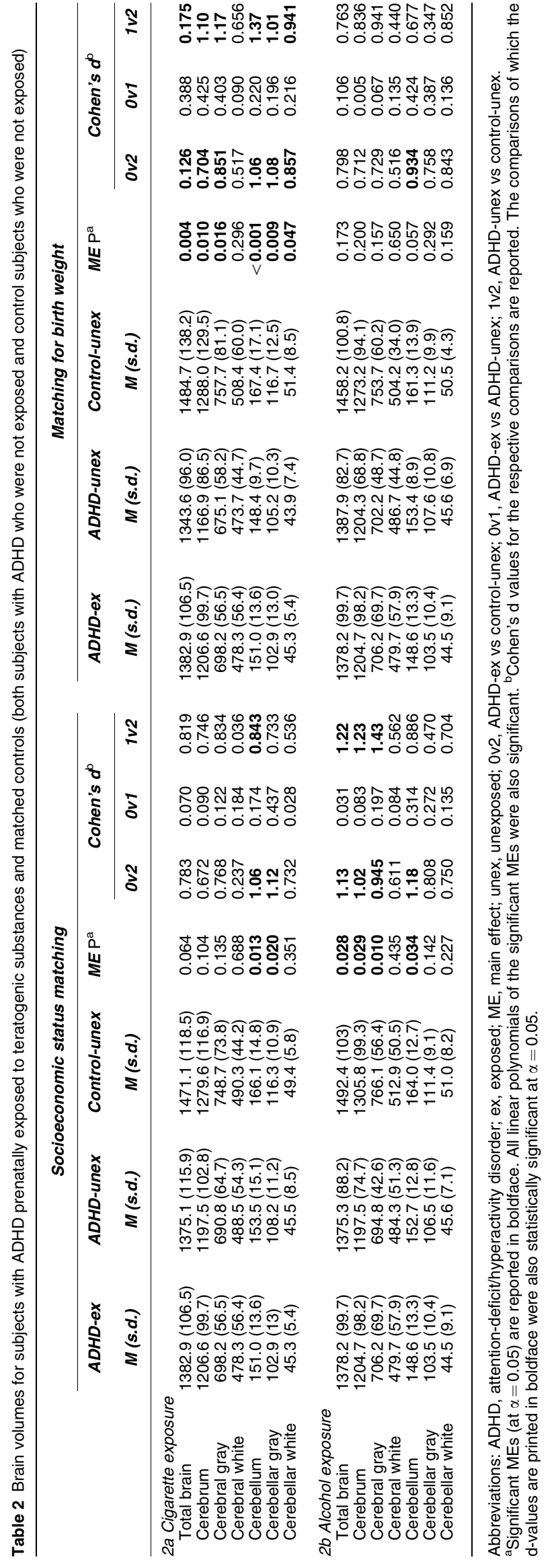

exposure to alcohol, we found main effects on cerebral volume and cerebral GM and cerebellum, with again the most pronounced graded effect on cerebellum. However, this graded effect was less clear than for cigarette exposure. Together, our results suggest that the neurobiological mechanisms involved in translating prenatal exposure to alcohol or cigarettes into a risk factor for developing ADHD may involve cerebellum. ${ }^{1,7}$

One important question that arises from the recent literature on prenatal exposure and ADHD,,$^{4,28,51-55}$ is whether shared genetic effects can explain both the use of cigarettes or alcohol during pregnancy and genetic transmission of ADHD. Although we have no measures to directly address this hypothesis, we did assess the rate of psychiatric morbidity in first-degree relatives of our subjects with $A D H D$. If $G \times E$ correlation or pleiotropy were indeed mediating these effects, we would expect an increase in this rate for prenatally exposed subjects with ADHD. We found no evidence of such an increase. In addition, the staircase pattern of our results for cerebellum volume suggests that both genetic and environmental effects may be affecting the brain phenotype, with prenatally exposed subjects with ADHD taking a 'double hit'. Finally, we have previously shown that cerebellum volume is reduced in children with ADHD but not in their unaffected siblings, which also suggests that non-familial factors are relevant to this brain region in ADHD. ${ }^{56}$

Notably, we found no effect of prenatal exposure to alcohol on brain volume in controls. Combined with recent findings that ADHD is more likely to be diagnosed in the children of parents with alcohol dependence, ${ }^{54}$ this could be taken to suggest that the effect of prenatal alcohol exposure on brain volume is likely to be more genetically mediated than that of cigarette exposure. However, the magnitude of alcohol exposure in our sample was generally low in comparison with the extant literature, suggesting that a dosage effect may also be in play.

The relevance of prenatal factors for adult disease is addressed in the developmental origins of health and disease hypothesis. ${ }^{57,58}$ One element of this hypothesis is that in the event of adverse prenatal circumstances, brain tissue may be spared relative to other tissues, such as muscle, ${ }^{59}$ and general metabolic programming and that this may be evidenced by such factors as body composition later in life. ${ }^{60}$ In our data, we find no evidence for effects of prenatal exposure to teratogenic substances on body mass index, either at birth or at the time of scanning. This does not seem to support the brain-sparing hypothesis. However, psychiatric phenotypes are increasingly becoming a focus of the developmental origins of health and disease, where epigenetic effects of prenatal exposure have been put forward as a candidate mechanism of action. ${ }^{12,58}$ Our data may be consistent with such an interpretation, as they suggest both genetic and environmental effects on the brain.

We repeated all our analyses on a data set matched for BW rather than SES to test whether our results were specific to prenatal nicotine or alcohol exposure or rather more generally related to a suboptimal prenatal environment. ${ }^{12,61}$ Although we felt that this analysis was informative enough to pursue, it should be noted its interpretation is complicated by the fact that prenatal exposure to alcohol or nicotine are both related 
Table 3 Brain volumes for controls who had or had not been prenatally exposed to teratogenic substances

\begin{tabular}{|c|c|c|c|c|c|c|}
\hline \multirow[t]{2}{*}{ Volume (mI) } & \multicolumn{3}{|c|}{ Socioeconomic status matching } & \multicolumn{3}{|c|}{ Birth weight matching } \\
\hline & $\begin{array}{c}\text { Control exposed } \\
M(\text { s.d.) }\end{array}$ & $\begin{array}{c}\text { Control unexposed } \\
\text { M (s.d.) }\end{array}$ & Cohen's d & $\begin{array}{c}\text { Control exposed } \\
M(\text { s.d.) }\end{array}$ & $\begin{array}{c}\text { Control unexposed } \\
\text { M (s.d.) }\end{array}$ & Cohen's d \\
\hline \multicolumn{7}{|c|}{ 3a Cigarette exposure } \\
\hline Total brain & $1398.3(124.7)$ & $1497.0(161.6)$ & 0.684 & $1398.3(124.7)$ & $1472.4(152.7)$ & 0.532 \\
\hline Cerebrum & 1212.5 (109.6) & $1309.0(147.9)$ & 0.741 & 1212.5 (109.6) & 1286.8 (136.5) & 0.600 \\
\hline Cerebral gray & 722.7 (67.0) & 772.2 (88.3) & 0.632 & $722.7(67.0)$ & $759.0(84.8)$ & 0.475 \\
\hline Cerebral white & $472.4(54.1)$ & $511.7(70.6)$ & 0.625 & $472.4(54.1)$ & $501.6(63.6)$ & 0.495 \\
\hline Cerebellum & $154.2(16.7)$ & $160.1(14.1)$ & 0.382 & $154.2(16.7)$ & $160.9(16.0)$ & 0.410 \\
\hline Cerebellar gray & $111.7(14.1)$ & $110.9(9.9)$ & 0.066 & $111.7(14.1)$ & $111.4(9.9)$ & 0.025 \\
\hline Cerebellar white & $42.4(6.9)$ & $48.5(7.6)$ & 0.840 & $42.4(6.9)$ & $48.1(8.4)$ & 0.742 \\
\hline \multicolumn{7}{|l|}{ 3b Alcohol exposure } \\
\hline Total brain & $1378.1(140.8)$ & 1397.9 (163.5) & 0.130 & $1378.1(140.8)$ & 1390.4 (141.2) & 0.087 \\
\hline Cerebrum & 1208.4 (129.5) & $1217.9(145.0)$ & 0.069 & $1208.4(129.5)$ & $1215.0(131)$ & 0.051 \\
\hline Cerebral gray & 723.1 (87.9) & 735.7 (84.4) & 0.146 & 723.1 (87.9) & $729.0(69.4)$ & 0.075 \\
\hline Cerebral white & $459.3(55.1)$ & $475.3(61.8)$ & 0.273 & $459.3(55.1)$ & $479.0(61.9)$ & 0.336 \\
\hline Cerebellum & $150.2(12.2)$ & $152.5(20.3)$ & 0.137 & $150.2(12.2)$ & $151.1(18.8)$ & 0.057 \\
\hline Cerebellar gray & $106.0(12.1)$ & $109.7(14.3)$ & 0.279 & $106.0(12.1)$ & 106.3 (13.9) & 0.023 \\
\hline Cerebellar white & $43.0(7.1)$ & $44.1(6.8)$ & 0.158 & $43.0(7.1)$ & $45.6(6.8)$ & 0.374 \\
\hline
\end{tabular}
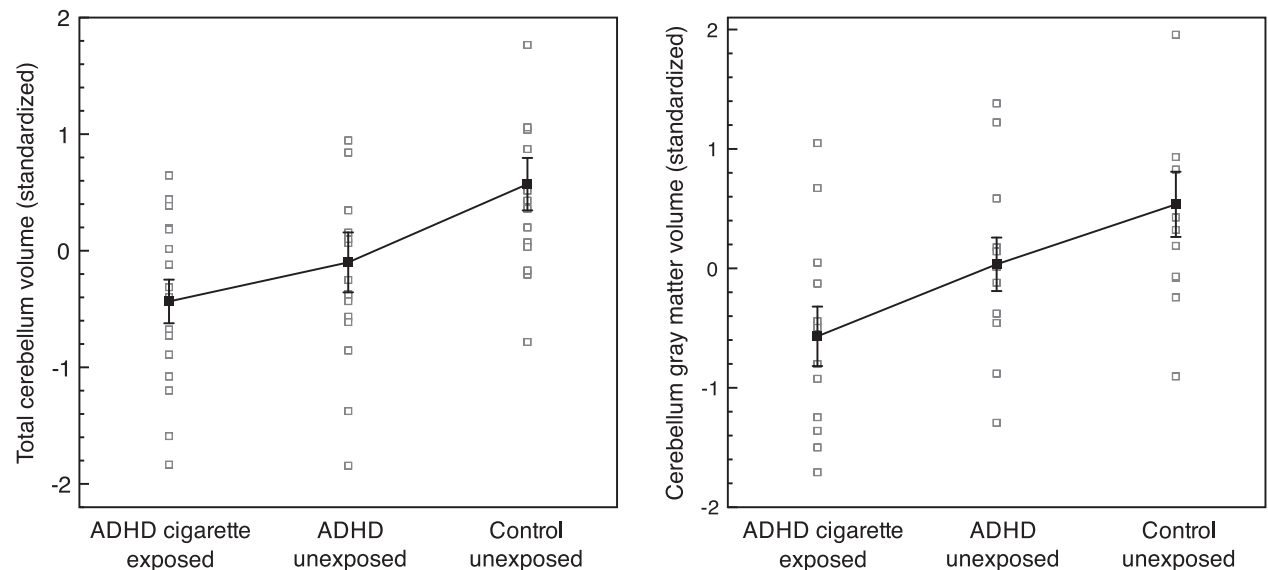

Figure 2 The effect of prenatal exposure to cigarettes is most pronounced in cerebellum. Cerebellum and cerebellum gray matter volume for subjects with attention-deficit/ hyperactivity disorder (ADHD) who were prenatally exposed to cigarette smoking, subjects with ADHD who were not and unexposed typically developing control subjects. Standardized data, residualized for the covariates in the analysis of variance are shown in gray, with group means and s.e. in black.
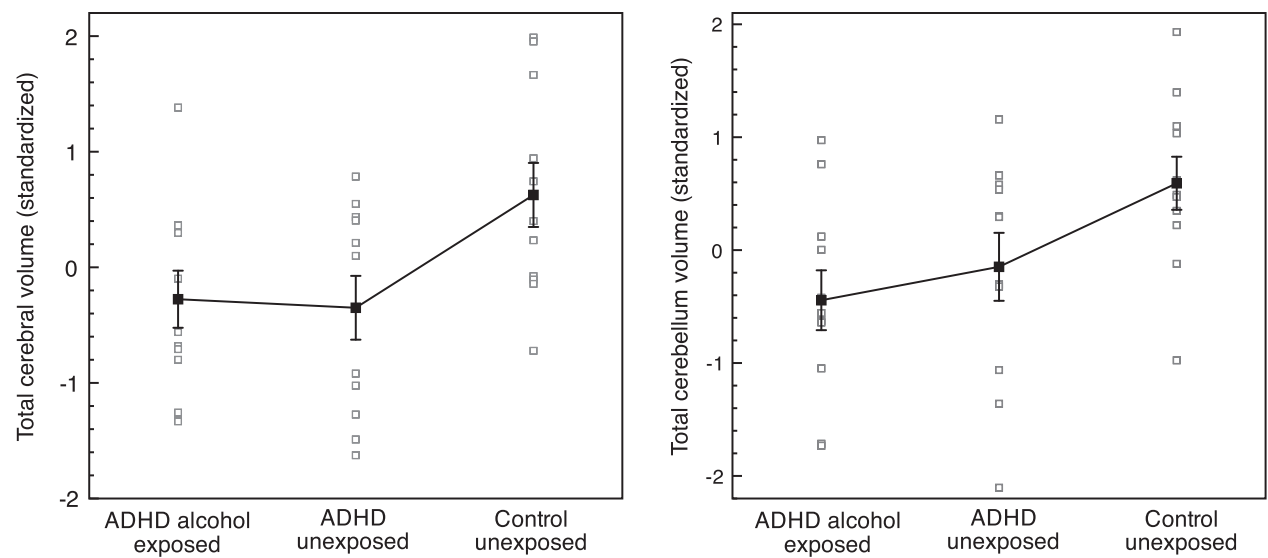

Figure 3 The effect of prenatal exposure to alcohol is most pronounced in cerebellum. Cerebral and cerebellum volume in subjects with attention-deficit/hyperactivity disorder (ADHD) who were prenatally exposed to alcohol, subjects with ADHD who were not and unexposed control subjects. Standardized data, residualized for the covariates in the analysis of variance are shown in gray, with group means and s.e. in black. 
to reduced $\mathrm{BW} .^{3,11}$ As such, by matching for BW we may in fact be overcorrecting for a variable of interest. In addition, matching for SES may also have provided more ecologically valid control over other factors affecting BW such as maternal nutrition. ${ }^{35}$ The general pattern of results in the analyses with data matched by BW was similar to that found in the analyses for groups matched by SES. For cigarette exposure, the brain volumes of exposed subjects with ADHD now resembled those of unexposed subjects with ADHD more closely. However, cerebellum GM volume still showed the staircase pattern of results. For alcohol exposure, none of the main effects found for groups matched for SES met significance, although a suggestive trend for cerebellum volume remained. This strengthened our conclusion that, similar to cigarette exposure, the most pronounced effect of alcohol exposure was on the cerebellum. In sum, these analyses show that whereas some of the effects of prenatal exposure to cigarettes and alcohol may be aspecific, there also appears to be a direct effect of exposure on brain volume, in particular on the volume of cerebellum.

There are some further limitations associated with our study. First, it is a naturalistic retrospective study of modest size. Furthermore, retrospective reports of substance use should always be considered with a certain level of caution. However, research has shown that maternal reports on the use of teratogenic substances during pregnancy are generally reliable, especially when dichotomized, as was done here. ${ }^{62}$ Importantly, it was impossible for us to control for mediating factors such as maternal nutritional status, timing of use and dosage or postnatal developmental environment in a naturalistic study. ${ }^{10,11}$ Therefore, we chose to use SES as a primary matching criterion. SES is often correlated with these confounding factors, offering some control over these issues. By repeating all analyses while controlling for BW, we were able to further address the specificity of our findings.

In conclusion, our results show that prenatal exposure to alcohol or nicotine is relevant to the volume of cerebellum. Our results are consistent with separate effects of prenatal exposure and ADHD on cerebellum volume. As such, they suggest that in ADHD, there may be an effect of prenatal exposure per se that cannot be fully explained by pleiotropic genetic effects.

\section{Conflict of interest}

Professor Dr Durston has received a research grant from Unilever Foods. The remaining authors declare no conflict of interest.

Acknowledgements. We thank the parents and children who participated in this study. This work was supported by VIDI Grant 91.776 .384 from the Netherlands Organization for Scientific Research (NWO) to SD.

1. Ernst M, Moolchan ET, Robinson ML. Behavioral and neural consequences of prenatal exposure to nicotine. J Am Acad Child Adolesc Psychiatry 2001; 40: 630-641.

2. O'Malley KD, Nanson J. Clinical implications of a link between fetal alcohol spectrum disorder and attention-deficit hyperactivity disorder. Can J Psychiatry 2002; 47: 349-354.

3. Huizink AC, Mulder EJ. Maternal smoking, drinking or cannabis use during pregnancy and neurobehavioral and cognitive functioning in human offspring. Neurosci Biobehav Rev 2006; 30: 24-41.
4. Knopik VS. Maternal smoking during pregnancy and child outcomes: real or spurious effect? Dev Neuropsychol 2009; 34: 1-36.

5. Linnet KM, Dalsgaard S, Obel C, Wisborg K, Henriksen TB, Rodriguez A et al. Maternal lifestyle factors in pregnancy risk of attention deficit hyperactivity disorder and associated behaviors: review of the current evidence. Am J Psychiatry 2003; 160: 1028-1040.

6. Orlebeke JF, Knol DL, Verhulst FC. Child behavior problems increased by maternal smoking during pregnancy. Arch Environ Health 1999; 54: 15-19.

7. Banerjee TD, Middleton F, Faraone SV. Environmental risk factors for attention-deficit hyperactivity disorder. Acta Paediatr 2007; 96: 1269-1274.

8. Taylor A, Warner Rogers J. Practitioner Review: early adversity and developmental disorders. J Child Psychol Psychiatry 2005; 46: 451-467.

9. Dwyer JB, McQuown SC, Leslie FM. The dynamic effects of nicotine on the developing brain. Pharmacol Ther 2009; 122: 125-139.

10. Levitt P. Prenatal effects of drugs of abuse on brain development. Drug Alcohol Depend 1998; 51: 109-125.

11. Thompson BL, Levitt $P$, Stanwood GD. Prenatal exposure to drugs: effects on brain development and implications for policy and education. Nat Rev Neurosci2009; 10: 303-312.

12. Mill J, Petronis A. Pre- and peri-natal environmental risks for attention-deficit hyperactivity disorder (ADHD): the potential role of epigenetic processes in mediating susceptibility. J Child Psychol Psychiatry 2008; 49: 1020-1030.

13. Derauf C, Kekatpure M, Neyzi N, Lester B, Kosofsky B. Neuroimaging of children following prenatal drug exposure. Semin Cell Dev Biol 2009; 20: 441-454.

14. Goodlett CR, Horn KH, Zhou FC. Alcohol teratogenesis: mechanisms of damage and strategies for intervention. Exp Biol Med (Maywood) 2005; 230: 394-406.

15. Hellstrom-Lindahl E, Gorbounova O, Seiger A, Mousavi M, Nordberg A. Regional distribution of nicotinic receptors during prenatal development of human brain and spinal cord. Brain Res Dev Brain Res 1998; 108: 147-160.

16. Guerri $C$. Mechanisms involved in central nervous system dysfunctions induced by prenatal ethanol exposure. Neurotox Res 2002; 4: 327-335.

17. Lebel C, Roussotte F, Sowell ER. Imaging the impact of prenatal alcohol exposure on the structure of the developing human brain. Neuropsychol Rev 2011; 21: 102-118.

18. Ikonomidou C, Bittigau P, Ishimaru MJ, Wozniak DF, Koch C, Genz K et al. Ethanolinduced apoptotic neurodegeneration and fetal alcohol syndrome. Science 2000; 287: 1056-1060.

19. Tiemeier H, Lenroot RK, Greenstein DK, Tran L, Pierson R, Giedd JN. Cerebellum development during childhood and adolescence: a longitudinal morphometric MRI study. Neurolmage 2010; 49: 63-70.

20. Roza SJ, Verburg BO, Jaddoe VW, Hofman A, Mackenbach JP, Steegers EA et al. Effects of maternal smoking in pregnancy on prenatal brain development. The Generation $R$ Study. Eur J Neurosci 2007; 25: 611-617.

21. Jaatinen $P$, Rintala J. Mechanisms of ethanol-induced degeneration in the developing, mature, and aging cerebellum. Cerebellum 2008; 7: 332-347.

22. Sowell ER, Jernigan TL, Mattson SN, Riley EP, Sobel DF, Jones KL. Abnormal development of the cerebellar vermis in children prenatally exposed to alcohol: size reduction in lobules I-V. Alcohol Clin Exp Res 1996; 20: 31-34.

23. Durston $S$, de Zeeuw $P$, Staal WG. Imaging genetics in ADHD: a focus on cognitive control. Neurosci Biobehav Rev 2009; 33: 674-689.

24. Valera EM, Faraone SV, Murray KE, Seidman LJ. Meta-analysis of structural imaging findings in attention-deficit/hyperactivity disorder. Biol Psychiatry 2007; 61: 1361-1369.

25. Paus T, Nawazkhan I, Leonard G, Perron M, Pike GB, Pitiot A et al. Corpus callosum in adolescent offspring exposed prenatally to maternal cigarette smoking. Neurolmage 2008; 40: 435-441.

26. Rivkin MJ, Davis PE, Lemaster JL, Cabral HJ, Warfield SK, Mulkern RV et al. Volumetric MRI study of brain in children with intrauterine exposure to cocaine, alcohol, tobacco, and marijuana. Pediatrics 2008; 121: 741-750.

27. Toro R, Leonard G, Lerner JV, Lerner RM, Perron M, Pike GB et al. Prenatal exposure to maternal cigarette smoking and the adolescent cerebral cortex. Neuropsychopharmacology 2008; 33: 1019-1027.

28. Knopik VS, Heath AC, Jacob T, Slutske WS, Bucholz KK, Madden PA et al. Maternal alcohol use disorder and offspring ADHD: disentangling genetic and environmental effects using a children-of-twins design. Psychol Med 2006; 36: 1461-1471.

29. Thapar A, Rice F, Hay D, Boivin J, Langley K, van den Bree M et al. Prenatal smoking might not cause attention-deficit/hyperactivity disorder: evidence from a novel design. Biol Psychiatry 2009; 66: 722-727.

30. Nigg J, Nikolas M, Burt SA. Measured gene-by-environment interaction in relation to attention-deficit/hyperactivity disorder. J Am Acad Child Adolesc Psychiatry 2010; 49: 863-873.

31. Shaffer D, Fisher $P$, Lucas CP, Dulcan MK, Schwab-Stone ME. NIMH Diagnostic Interview Schedule for Children Version IV (NIMH DISC-IV): description, differences from previous versions, and reliability of some common diagnoses. J Am Acad Child Adolesc Psychiatry 2000; 39: 28-38.

32. Verhulst F, Van Der Ende J, Koot $\mathrm{H}$. Handleiding voor de CBCL/4-18 (Manual for the CBCL/4-18). Department of Child and Adolescent Psychiatry, Erasmus Academic Medical Centre: Rotterdam, 1996.

33. Wechsler D. Wechsler Intelligence Scale for Children - Derde Editie NL. Handleiding en Verantwoording. (Wechsler Intelligence Scale for Children - Third Edition, Dutch Version, Manual). Harcourt Assessment: London, 2005. 
34. Hackman DA, Farah MJ, Meaney MJ. Socioeconomic status and the brain: mechanistic insights from human and animal research. Nat Rev Neurosci 2010; 11: 651-659.

35. Abu-Saad K, Fraser D. Maternal nutrition and birth outcomes. Epidemiol Rev 2010; 32 $5-25$

36. Durston S, Fossella JA, Casey BJ, Hulshoff Pol HE, Galvan A, Schnack HG et al Differential effects of DRD4 and DAT1 genotype on fronto-striatal gray matter volumes in a sample of subjects with attention deficit hyperactivity disorder, their unaffected siblings, and controls. Mol Psychiatry 2005; 10: 678.

37. De Zeeuw $P$, Schnack $H$, Van Belle J, Weusten JM, van Dijk SC, Langen $M$ et al. Differential brain development with low and high IQ in attention-deficit/hyperactivity disorder. (submitted).

38. Hulshoff Pol HE, Schnack HG, Posthuma D, Mandl RC, Baare WF, van OC et al. Genetic contributions to human brain morphology and intelligence. J Neurosci 2006; 26: 10235.

39. Langen M, Schnack HG, Nederveen H, Bos D, Lahuis BE, de Jonge MV et al. Changes in the developmental trajectories of striatum in autism. Biol Psychiatry 2009; 66: 327-333.

40. Talairach J, Tournoux P. Co-planar Stereotaxic Atlas of the Human Brain: 3-Dimensional Proportional System: An Approach to Cerebral Imaging. Thieme: New York, 1988.

41. Maes F, Collignon A, Vandermeulen D, Marchal G, Suetens P. Multimodality image registration by maximization of mutual information. IEEE Trans Med Imag 1997; 16: 187-198.

42. Sled JG, Zijdenbos AP, Evans AC. A nonparametric method for automatic correction of intensity nonuniformity in MRI data. IEEE Trans Med Imag 1998; 17: 87-97.

43. Schnack HG, Hulshoff HE, Baare WF, Viergever MA, Kahn RS. Automatic segmentation of the ventricular system from MR images of the human brain. Neurolmage 2001 14(1 Part 1): 95-104.

44. Schnack HG, Hulshoff Pol HE, Baare WF, Staal WG, Viergever MA, Kahn RS. Automated separation of gray and white matter from MR images of the human brain. Neurolmage 2001; 13: 230-237.

45. Brouwer RM, Hulshoff Pol HE, Schnack HG. Segmentation of MRI brain scans using non-uniform partial volume densities. Neurolmage 2010; 49: 467-477.

46. Giedd JN, Lalonde FM, Celano MJ, White SL, Wallace GL, Lee NR et al. Anatomica brain magnetic resonance imaging of typically developing children and adolescents. J Am Acad Child Adolesc Psychiatry 2009; 48: 465-470.

47. Lenroot RK, Gogtay N, Greenstein DK, Wells EM, Wallace GL, Clasen LS et al. Sexual dimorphism of brain developmental trajectories during childhood and adolescence. Neurolmage 2007; 36: 1065-1073.

48. Shaw P, Kabani NJ, Lerch JP, Eckstrand $K$, Lenroot $R$, Gogtay $N$ et al. Neurodevelopmental trajectories of the human cerebral cortex. J Neurosci 2008; 28: 3586-3594.

49. Sowell ER, Thompson PM, Leonard CM, Welcome SE, Kan E, Toga AW. Longitudinal mapping of cortical thickness and brain growth in normal children. $J$ Neurosci 2004; 24 8223-8231.
50. Ostby Y, Tamnes CK, Fjell AM, Westlye LT, Due-Tonnessen P, Walhovd KB. Heterogeneity in subcortical brain development: a structural magnetic resonance imaging study of brain maturation from 8 to 30 years. J Neurosci 2009; 29: 11772-11782.

51. Thapar A, Fowler T, Rice F, Scourfield J, van den Bree M, Thomas $\mathrm{H}$ et al. Maternal smoking during pregnancy and attention deficit hyperactivity disorder symptoms in offspring. Am J Psychiatry 2003; 160: 1985-1989.

52. Button TM, Thapar A, McGuffin P. Relationship between antisocial behaviour, attentiondeficit hyperactivity disorder and maternal prenatal smoking. Br J Psychiatry 2005; 187 : 155-160.

53. Agrawal A, Scherrer JF, Grant JD, Sartor CE, Pergadia ML, Duncan AE et al. The effects of maternal smoking during pregnancy on offspring outcomes. Prev Med 2010; 50: 13-18.

54. Knopik VS, Sparrow EP, Madden PA, Bucholz KK, Hudziak JJ, Reich W et al. Contributions of parental alcoholism, prenatal substance exposure, and genetic transmission to child ADHD risk: a female twin study. Psychol Med 2005; 35: 625-635.

55. Maughan B, Taylor A, Caspi A, Moffitt TE. Prenatal smoking and early childhood conduct problems: testing genetic and environmental explanations of the association. Arch Gen Psychiatry 2004; 61: 836-843.

56. Durston S, Hulshoff Pol HE, Schnack HG, Buitelaar JK, Steenhuis MP, Minderaa RB et al. Magnetic resonance imaging of boys with attention-deficit/hyperactivity disorder and their unaffected siblings. J Am Acad Child Adolesc Psychiatry 2004; 43: 332-340.

57. Barker DJ. The origins of the developmental origins theory. $J$ Intern Med 2007; 261: 412-417.

58. Gillman MW, Barker D, Bier D, Cagampang F, Challis J, Fall C et al. Meeting report on the 3rd International Congress on Developmental Origins of Health and Disease (DOHaD). Pediatr Res 2007; 61(5 Part 1): 625-629.

59. Baker J, Workman M, Bedrick E, Frey MA, Hurtado M, Pearson O. Brains versus brawn: an empirical test of Barker's brain sparing model. Am J Hum Biol 2010; 22: 206-215.

60. Oken E, Levitan EB, Gillman MW. Maternal smoking during pregnancy and child overweight: systematic review and meta-analysis. Int J Obes (Lond) 2008; 32: 201-210.

61. Nigg JT, Breslau N. Prenatal smoking exposure, low birth weight, and disruptive behavior disorders. J Am Acad Child Adolesc Psychiatry 2007; 46: 362-369.

62. Pickett KE, Kasza K, Biesecker G, Wright RJ, Wakschlag LS. Women who remember, women who do not: a methodological study of maternal recall of smoking in pregnancy. Nicotine Tob Res 2009; 11: 1166-1174.

Translational Psychiatry is an open-access journal published by Nature Publishing Group. This work is licensed under the Creative Commons Attribution-Noncommercial-No Derivative Works 3.0 Unported License. To view a copy of this license, visit http://creativecommons.org/licenses/by-nc-nd/3.0/

Supplementary Information accompanies the paper on the Translational Psychiatry website (http://www.nature.com/tp) 